\title{
Tumor markers for hepatocellular carcinoma (Review)
}

\author{
YAN-JIE ZHAO, QIANG JU and GUAN-CHENG LI \\ Tumor Immunobiology Laboratory of Cancer Research Institute, Key Laboratory of Carcinogenesis \\ and Cancer Invasion Ministry of Education, Key Laboratory of Carcinogenesis Ministry of Health, \\ Central South University, Changsha, Hunan 410078, P.R. China
}

Received September 25, 2012; Accepted April 15, 2013

DOI: $10.3892 / \mathrm{mco} .2013 .119$

\begin{abstract}
Hepatocellular carcinoma (HCC) is one of the most common malignant tumors with a high rate of morbidity and mortality. HCC affects approximately one million individuals annually worldwide, with the incidence equal to the mortality rate. In 2008, HCC was listed as the third most lethal cancer. Thus, early diagnosis is crucial for improving the survival rate for patients. $\alpha$-fetoprotein (AFP) together with iconography and pathology detection are commonly used in the clinical early diagnosis of liver cancer. However, the specificity and sensitivity of AFP used in screening for liver cancer are not satisfactory. Athough the development of molecular biology has led to the identification of new tumor markers, including proteantigens, cytokines, enzymes and isoenzymes, as well as related genes that can be used in the treatment and prognosis of liver cancer, more tumor markers are required for effective early diagnosis of diseases and monitoring of the curative effect.
\end{abstract}

\section{Contents}

1. Introduction

2. Embryonic antigen

3. Proteantigen

4. Enzymes and isozymes

5. Cytokines

6. Genetic biomarkers

7. Conclusions

Correspondence to: Professor Guan-Cheng Li, Cancer Research Institute, Key Laboratory of Carcinogenesis and Cancer Invasion Ministry of Education, Key Laboratory of Carcinogenesis Ministry of Health, Central South University, Changsha, Hunan 410078, P.R. China

E-mail: libsun@163.com

Key words: hepatocellular carcinoma, early diagnosis, tumor markers

\section{Introduction}

Primary hepatic cancer (PHC) is one of the most common malignant tumors with $90-95 \%$ of liver cancer being hepatocellular carcinoma (HCC). Liver cancer exhibits no symptoms in the early stage, whereas clinical symptoms are evident in the advanced stage, leading to an unsatisfactory curative effect. In 2008, HCC was listed as the third most lethal cancer type (1). Findings of a previous study suggested that early diagnosis of HCC and effective treatment are likely to prolong the lifetime of liver cancer patients (2). Thus, the identification of new high sensitivity and specificity markers for $\mathrm{HCC}$ are essential.

$\alpha$-fetoprotein (AFP) together with iconography and pathology detection are commonly used in clinical early diagnosis for liver cancer. However, the widely used marker AFP does not yield satisfactory results in the early diagnosis of HCC, particularly AFP-negative HCC. These false-negative results limit the universality of its application. In recent years, the development of molecular biology has led to the successful exploration and identification of markers for $\mathrm{HCC}$, which is expected to improve the early diagnostic rate, treatment effect in addition to curative satisfaction.

\section{Embryonic antigen}

$A F P$. AFP is the most widely used tumor biomarker currently available for the early detection of HCC. Findings of a previous clinical study demonstrated that serum AFP had a sensitivity of $41-65 \%$ and specificity of $80-94 \%$ when the cut-off value is $20 \mathrm{ng} / \mathrm{ml}$ (3). However, the following issues should be considered with regard to early diagnosis: i) the positive rate of AFP in HCC is only $60-80 \%$, making it a limitation; ii) false-positive, AFP results are positive during pregnancy, as well as for active liver disease, embryonic tumor and certain gastrointestinal tumors; iii) false-negative, limitations in terms of sensitivity in different detection methods. Additionally, a small hepatic tumor results in AFP expression being lower than the limit of detection, whereas AFP expression is delayed or higher than the limit of detection when the tumor is large, yielding AFP-negative HCC.

AFP heterogeneity. The development and applications of biological chemistry and related analysis, as well as additional study of AFP have revealed that AFP has three glycoforms 
(AFP-L1, AFP-L2 and AFP-L3), according to their binding ability to the lectin lens agglutinin (LCA). AFP-L1, as a nonLCA-bound heterogeneity, is a major glycoform in various benign liver diseases. By contrast, AFP-L3, as an LCA-bound heterogeneity, exists only in the serum of patients with HCC at a cut-off value of $15 \%$, with a sensitivity and specificity of 96.9 and $92 \%$, respectively, in detecting HCC (4). The sensitivity and specificity of AFP-13 are both relatively satisfactory as compared with AFP. Moreover, AFP-L3 does not correlate with AFP, thus the former can be used as an independent and significant factor for the early diagnosis of HCC. Specifically, for HCC patients with a total AFP of 10-200 $\mathrm{ng} / \mathrm{ml}$, when the cut-off value of AFP-L3 is 35\%, the diagnostic specificity for HCC reaches $100 \%$, thereby improving the early diagnostic rate (5). Using the $\mu$ TAS method, ALP-L3\% was detected with high sensitivity in the diagnosis of $\mathrm{HCC}$ at stage I or when the tumor size was $<2 \mathrm{~cm}(42.5$ and $46.0 \%$, respectively). When the cut-off value of AFP-L3\% is 5\%, the sensitivity for HCC reaches $47.2 \%$ compared with that of $38 \%$ for total AFP (6). Consequently, AFP-L3 is expected to be a useful marker for HCC.

\section{Proteantigen}

$H S P$. Heat shock protein (HSP) is a highly conserved stress response protein. It can protect cells and promote them to repair the damage caused by a variety of stimuli. HSP is expressed under physiological and stress conditions, including carcinogenesis. By immunohistochemical staining, Joo et al (7) identified the positive rate of HSP70 and HSP27 to be 56.3 and $61.9 \%$, respectively, in HCC tissues. The stained intensity of HSP70 was positively correlated with tumor size, portal vein invasion and tumor stage, while HSP27 was only associated with $\mathrm{HCC}$ which are infected by hepatitis B virus (HBV). In the microenvironment of hepatocarcinoma, the overexpression of HSP70 and HSP27 was able to promote tumor growth and metastasis (8).

HSP70 may be used as an indicator of prognosis for HCC. Its expression was observed in 282 of 392 HCC cases (71.9\%), whereas only 14 of 115 non-neoplastic liver tissues expressed HSP70 $(\mathrm{P}<0.001)(9)$. The sensitivity and specificity in detecting HCC were identified as 57.5 and $85 \%$, respectively (10). Furthermore, the expression of HSP70 is correlated with differentiation and apoptosis of tumor cells. It promotes tumor cell growth by stabilizing cyclin D1 and suppresses the apoptosis of tumor cells by inhibiting the p53 pathway $(11,12)$. Thus, HSP70 and HSP27 are potential markers for HCC and should be further investigated.

Glypican-3 (GPC3). GPC3 is a family of the heparan sulfate proteoglycans that is linked to the cell membrane by a glycosylphosphatidylinositol (GPI) anchor (13). GPC3 is involved in the process of regulating cell growth, development, differentiation and migration. sGPC3, which lack GPI, inhibit the growth of HCC by removing several pro-tumorigenic growth factors including Wnts, hepatocyte growth factor (HGF) and vascular endothelial growth factor (VEGF) from the surface of HCC cells in vitro and in vivo (14). The expression of GPC3 was upregulated in HCC tumor tissues compared with normal and benign liver diseases and contributed to promoting the growth of $\mathrm{HCC}$ by stimulating Wnt signaling (15). In addition, no correlation between GPC 3 expression and tumor stage, size and AFP level has been observed. The sensitivity and specificity in the diagnosis of HCC was found to be 77 and $96 \%$, respectively (16). On the strength of these results, GPC3 is a potential marker for HCC.

SCCA. Squamous cell carcinoma antigen (SCCA), a serine protease inhibitor isolated from cervical carcinoma, is typically expressed in epithelial tumors and protects tumor cells from apoptosis. Guido et al found that the expression of SCCA in HCC (93\%) and dysplastic nodule (100\%) is significantly higher than the regenerative nodule (29\%), suggesting that the expression of SCCA increased in the early stages of HCC formation (17). In addition, high SCCA values in the tumor were inversely correlated with the nodule size (18), which had a sensitivity and specificity of 84 and $46 \%$, respectively, in detecting HCC (19). The high sensitivity and low specificity were complementary with AFP. Thus, SCCA was a valuable supplement marker for the diagnosis of HCC.

SCCA-IgM IC is a circulating immune complex composed of SCCA and IgM. It was undetectable in the sera of a healthy control population. However, in chronic hepatitis, cirrhosis and HCC, the detection rates of SCCA-IgM IC were 18, 26 and $70 \%$, respectively. No correlation was identified with AFP level (20). Furthermore, in patients with LC progressing towards HCC, SCCA-IgM IC was consistently increased and had higher sensitivity compared with AFP (21). Therefore, SCCA-IgM IC may be a novel valuable serum marker for HCC. A combination of SCCA-IgM IC and AFP can thus improve the diagnostic rate.

Golgi protein 73 (GP73). GP73 (also known as Golph2 and GOLM1) is a type II Golgi-specific membrane protein that is coded by the GOLM1 gene on chromosome 9q21.33 (22). It is significantly elevated in various types of cancer, such as lung adenocarcinoma (23), seminomas (24) and renal cell cancer (25). However, GP73, is closely associated with liver diseases, particularly $\mathrm{HCC}$, and has received increasing attention. Results of recent studies have shown that the serum GP73 is significantly elevated in primary hepatic carcinoma (PHC) $(26,27)$. In liver cirrhosis it is not only elevated, but also higher than in HCC, whether infection is caused by HBV or hepatitis C virus (HCV) (28). By contrast, in normal liver, GP73 is expressed by biliary epithelial cells, but minimally by hepatocytes (29). Thus, GP73 is a significant factor in many hepatic diseases. In their study, Mao et al (31) demonstrated that GP73 in the serum of patients with HCC infected by HBV was significantly higher compared with HBV carriers, patients without hepatic diseases and healthy adults. The sensitivity of diagnosis of HCC (76.9\%) was markedly elevated compared with AFP (48.6\%), suggesting GP73 is a novel and effective serum biomarker for the diagnosis of HCC $(30,31)$.

Additional investigations identified fucosylated GP73 (FC-GP73). Compared with total GP73, FC-GP73 improves the sensitivity and specificity of diagnosis of HCC from 65-90 to 90-100\%, respectively. For GP73-negative or low levels, detection of FC-GP73 is a viable option (32). Although the study for GP73 is optimistic, there are limitations that should be considered such as the fact that the correlation 
between GP73 and tumor size, stage, recurrence and prognosis should be extensively investigated. Specifically, the mechanism for GP73 and HCC development remains to be elucidated. Thus, role of GP73 in the clinic remains to be determined.

Tumor-associated glycoprotein 72 (TAG-72).TAG-72 is a macromolecular glycoprotein complex similar to mucin-1 (MUC-1). It is overexpressed in the majority of human adenocarcinomas including gastric, colon and pancreatic cancer. However, it is rarely expressed in normal tissues. Recent studies (33) found that the expression of TAG-72 is significantly elevated in HCC tissues compared with normal liver tissues. Its increased expression may promote tumor invasion and metastasis. Furthermore, overexpression of TAG-72 is closely correlated with poor survival in patients with HCC (33). Thus, TAG-72 is a potential prognostic marker for HCC, which has important clinical implications. Moreover, anti-TAG-72 monoclonal antibody has been used for the clinical detection of tumors (34).

Zinc- $\alpha$ 2-glycoprotein (ZAG). ZAG, a soluble glycoprotein, is a member of the class I major histocompatibility complex (MHC-I) family. Due to its high homology of the amino acid sequence with the lipid mobilizing factor (LMF), we considered it a novel adipokine. ZAG is downregulated in human obesity (35). By contrast, it is upregulated in several types of cancer such as breast, lung and prostate cancer and is considered as a potential biomarker for these cancer types. By analyzing the serum proteome of the HCC group, liver cirrhosis group and healthy adults, we confirmed that the ZAG protein is overexpressed in the HCC group, suggesting it is a novel candidate biomarker for the early diagnosis of HCC (36).

\section{Enzymes and isozymes}

Des- $\gamma$-carboxyprothrombin $(D C P)$. DCP is an abnormal protein induced by the absence of vitamin $\mathrm{K}$. When the liver cells are in the process of malignant transformation, an obstacle occurs for the vitamin K-dependent carboxylation system. Insufficient reaction of $\gamma$-glutamyl carboxylation causes the production of DCP (37). The level of DCP in the serum of patients with HCC was significantly higher than that in patients with chronic hepatitis and cirrhosis. The diagnostic sensitivity of DCP was weaker compared with AFP when $\mathrm{HCC}$ was $<3 \mathrm{~cm}$ in diameter, while it was stronger compared with AFP when HCC was $>5 \mathrm{~cm}$ in diameter (38). However, Baek et al recently demonstrated that irrespective of whether the diameter of $\mathrm{HCC}$ is $<3 \mathrm{~cm}, 3-5 \mathrm{~cm}$ or $>5 \mathrm{~cm}$, the diagnostic accuracy of DCP was higher than that of AFP (39). In addition, the combined detection of DCP and AFP can improve the diagnostic sensitivity and can be used to predict the recurrence of HCC within 6 months after surgery (40). By contrast, the level of DCP is closely associated with a larger tumor, vascular invasion and it serves as a more accurate tumor marker compared with AFP and AFP-L3 (41).

$\gamma$-glutamyl transferase $(G G T)$. GGT, a plasma membranebound enzyme, is synthesized in the microsomes of human cells. It is highest in embryo livers and decreases rapidly to the lowest levels after birth (42). The activity of serum GGT is extremely low in healthy adults, but stimulations such as cholestasis and inflammation can improve the level of serum GGT to varying degrees. GGT mRNA is widely distributed in the liver tissues of healthy adults, patients with liver disease, benign liver tumor and HCC (4). The development of HCC due to cancer cell inverse differentiation is similar to the embryonic stage, improving the level of serum GGT to a moderate or high degree. Therefore, GGT may serve as a marker for HCC. However, the sensitivity of GGT to detect small HCC is only $43.8 \%$ (43). This enzyme is therefore only used to aid in the diagnosis of HCC. In addition, it has been previously shown that simultaneous determination of GGT, DCP and AFP can significantly improve the rate of diagnosis of HCC (44).

$\alpha$-l-fucosidase (AFU). AFU is a lysosomal enzyme widely present in human cells, blood and body fluid. It can be detected in the serum of healthy adults, although its activity increases in the serum of HCC patients. Additionally, it does not correlate with tumor size or the level of AFP (45). It has been reported that the sensitivity, specificity and diagnostic accuracy of AFU at the cut-off value of $2.3005 \mu \mathrm{mol} / 1 / \mathrm{min}$ were $90,97.5$ and $94.9 \%$, respectively. The combined detection of AFP and AFU can improve the sensitivity, specificity and diagnostic accuracy of AFP from 70, 85 and 79.7\%, respectively, to 95, 100 and $99.1 \%$, respectively (46). In addition, AFU can reveal the case 6-9 months earlier than ultrasonographic visualization (47). Thus, AFU may be a valuable supplementary marker in HCC detection. However, the specificity of AFU is relatively poor, and is also overexpressed in diabetes, pancreatitis and hypothyroidism patients. The activity of AFU is also susceptible to ethnicity. Therefore, the clinical value of AFU requires additional investigation.

\section{Cytokines}

Transforming growth factor- $\beta 1$ (TGF- $\beta 1)$. TGF- $\beta 1$ is a pluripotent growth factor involved in the regulation of cell proliferation, differentiation, embryo formation, angiogenesis, invasion and immune function. It is highly expressed in tumor cells, inhibiting the proliferation of tumor specific cytotoxic T lymphocytes (CTL) and NK cells, promoting the growth of tumor cells. The level of serum TGF- $\beta 1$ has been reported to be elevated in HCC patients compared with healthy adults or patients with non-malignant liver disease (48). It could induce microvascular abnormalities through the downregulation of neural cell adhesion molecules in human HCC (49). TGF- $\beta 1$ and TGF- $\beta 1$ mRNA may be used as sensitive indicators to diagnose $\mathrm{HCC}$ which is induced by HBV, with the sensitivity and specificity being 89.5 and $94.0 \%$, respectively, when TGF- $\beta 1$ is $>1.2 \mu \mathrm{g} / 1$ (50). The study of TGF- $\beta 1$ demonstrated that TGF- $\beta 1$ mediated different biological effects through different signaling pathways. The polymorphism of TGF- $\beta 1$ expression can affect susceptibility to tumor. TGF- $\beta 1$ signaling pathway as a tumor therapeutic target has become a hot spot.

$V E G F$. VEGF is well known to play a crucial role in tumor angiogenesis by inducing new vessel formation and promoting tumor invasion and metastasis. The expression of VEGF is higher in HCC patients. Xiang et al (51) revealed that VEGF is a type of biomarker of lymph node metastasis in HCC. In addition, the expression of VEGF is closely correlated with tumor recur- 
Table I. Markers for HCC.

\begin{tabular}{llccl}
\hline Markers & $\begin{array}{c}\text { The expression in the serum } \\
\text { /tissues of HCC }\end{array}$ & Sensitivity $(\%)$ & Specificity (\%) & \multicolumn{1}{c}{ Application } \\
\hline AFP & Upregulation & $41.0-65.0$ & $80.0-94.0$ & Early diagnosis \\
AFP-L3 & Upregulation & 96.9 & 92.0 & Early diagnosis \\
HSP70 & Upregulation & 57.5 & 85.0 & Prognosis \\
GPC3 & Upregulation & 77.0 & 96.0 & Diagnosis \\
SCCA & Upregulation & 84.0 & 46.0 & Early diagnosis \\
GP73 & Upregulation & 76.9 & - & Diagnosis \\
FC-GP73 & Upregulation & 90.0 & 100.0 & Diagnosis \\
GGT & Upregulation & 43.8 & - & Diagnosis \\
AFU & Upregulation & 90.0 & 97.5 & Diagnosis \\
AFU+AFP & Upregulation & 95.0 & 100.0 & Diagnosis \\
TGF- 31 & Upregulation & 89.5 & 94.0 & Prognosis \\
VEGF & Upregulation & - & - & Recurrence and prognosis \\
AFP-mRNA & Upregulation & - & - & Recurrence and prognosis \\
miR-21 & Upregulation & 87.3 & 92 & Diagnosis \\
miR-500 & Downregulation & - & - & Prognosis \\
miR-29 & Downregulation & - & - & Prognosis \\
miR-122 & Downregulation & - & - & Prognosis
\end{tabular}

HCC, hepatocellular carcinoma; AFP, $\alpha$-fetoprotein; HSP70, heat shock proteins 70; GPC3, Glypican-3; SCCA, squamous cell carcinoma antigen; FC-GP73, fucosylated GP73; GGT; $\gamma$-glutamyl transferase; AFU, $\alpha$-1-fucosidase; TGF- $\beta 1$, transforming growth factor- $\beta 1$; VEGF, endothelial growth factor; miR, miRNA.

rence and prognosis. Of note, some VEGF receptor expression has been found to correlate with the development of tumor (52). Knockdown of VEGF165 is able to inhibit the proliferation, migration and adhesion ability of HCC cells by increasing the expression of p53 signaling molecules (52). Therefore, the overexpression of VEGF may be useful as a biologic marker of tumor invasiveness and in predicting poor prognosis.

\section{Genetic biomarkers}

AFP $m R N A$. AFP mRNA is considered the most valuable marker for circulating HCC cells and is only present in active HCC cells. If other interferences such as genital tumors and peripheral blood are excluded, AFP mRNA could be used as a significant marker for spreading of HCC in blood. In advanced liver cancer, the rate of AFP mRNA expression reaches $100 \%$. Moreover, the value of AFP mRNA serves as a predictor for HCC recurrence. However, use of this marker is controversial, possibly due to the blood-borne dispersion of normal liver cells and tumor cells and the mis-transcription of mRNA encoding AFP by peripheral mononuclear cells (4). The positive rate of AFP mRNA in the recrudescent patients was $82.4 \%$, significantly higher compared with the group without recurrence (53). Thus, AFP mRNA effectively predicts tumor recurrence and metastasis following surgery.

MicroRNAs. MicroRNAs are small non-coding RNAs that effectively block translation by promoting the degradation of target mRNAs or binding to complementary sequences in the 3 ' untranslated region (UTR). In recent years, the association between microRNAs and tumors has becomes a point of debate.
MiR-500 (miRNA) is a potential candidate biomarker for HCC, as proven by Yamamoto et al (54), using a global miRNA expression profile in mouse liver development. This profile was highly expressed in embryo liver, downregulated in the process of liver development and then upregulated in the process of cirrhosis. Based on miRNA microarray, miR-29 was downregulated in HCC cells, suggesting its role as a prognostic marker for HCC therapy (55). Moreover, miR-199a/b-3p was also downregulated in HCC compared with normal liver and hepatitis liver, indicating its role as a prognostic target for HCC (56). MiR-122 is a liver-specific microRNA that is downregulated in HCC cells. Overexpression of miR-122 maintained cells in G2/M phase by regulating MDR expression (57). However, loss of miR-122 promoted HCC cell migration and invasion, rendering miR-122 a prognostic target for HCC.

An analysis of the miRNA signatures of a large number of tumor samples revealed that only miR-21 is upregulated in the tumors (58). Moreover, similar results have demonstrated that miR-21 is a central oncomiR (59). Plasma miR-21 level in patients with HCC was significantly higher than that in patients with chronic hepatitis and healthy individuals. It promoted HCC growth by regulating the expression of PENT and PENTrelated pathways (60). ROC analysis revealed the sensitivity and specificity to be 87.3 and $92 \%$, respectively, differentiating HCC patients from healthy adults. Thus, miR-21 is also a promising biomarker of HCC (61).

Related genes. $\Delta$-like 1 homolog (DLK1) is a newly identified hepatic stem/progenitor cell marker in fetal livers which is crucial in the oncogenesis of HCC. DLK1 is usually only expressed in embryonic, kidney and neuronal tissue. 
However, in HCC, DLK1 expression is significantly elevated and a similar phenomenon was also observed in small cell lung cancer, neuroblastoma and leukemia, suggesting that DLK1 is important in the occurrence of these tumors. Jin et al (62) reported that DLK1 expression does not correlate with AFP level and tumor metastasis, suggested DLK1 is an independent prognostic marker (62).

Hepatoma-associated gene (HTA) is a tumor differentially expressed gene obtained by bioinformatic analysis. Findings of a recent study showed that HTA is present only in tumors and absent in normal tissues. It is tumor-specific and the positive rate is extremely high in HCC in particular, suggesting a key role in the diagnosis and treatment of HCC (63).

Villin1 (Vil1) is a newly identified marker for HCC. The upregulation of Villin1 mRNA in high serum AFP-associated HCC tumor tissues induced poor differentiation, vascular invasion, advanced cancer stage and recurrence-free survival. Thus, Vil1 is a potential candidate molecular marker for high serum AFP-associated HCC (64).

\section{Conclusions}

Tumor progression is a complex disease process involving various factors, changes of various genes, and the development of multiple stages. During tumor progression, the number, species, distribution and expression level of tumor markers in patients with HCC exhibit variations that are closely associated with the occurrence, development, transfer, effect and prognosis of tumors. Large numbers of HCC markers exist in the clinical setting, however, most single indicators lack specificity of the tissues and organs. Furthermore, the single indicator results in the varying degrees of false positivity in certain benign diseases. Therefore, effective test strategies should be considered to improve the early diagnostic rate of HCC including: i) the combined detection of several serum markers that can complement each other in order to improve the early diagnostic rate. The sensitivity of AFP-L3, FC-GP73, AFU and SCCA is superior to that of AFP (Table I). Combined detection can increase the rate of diagnosis of $\mathrm{HCC}$ and reduce misdiagnosis. Other markers including GPC3 and TGF- $\beta 1$ have a very high specificity. Combined detection with AFP can significantly improve the ability of identification and diagnosis for HCC. ii) Selection of different indices based on different detection purposes: for example, AFP mRNA, TGF- $\beta 1$ and VEGF should be the first option in the prediction of HCC metastasis. iii) Diagnosis of HCC at the genetic level through the detection of the expression of AFP mRNA in peripheral blood, which is likely to improve the early diagnostic rate of HCC, as well as predict the transfer and recurrence of tumors. iv) The diagnosis of HCC must be combined with clinical manifestation, iconography detection and histological examination. Additional studies are likely to yield novel markers and adopt more effective combined detection methods to improve the sensitivity and specificity of diagnosis of $\mathrm{HCC}$, resulting in improved treatment and prognosis.

\section{Acknowledgements}

This study was supported by the National Natural Science Foundation of China (nos. 81201903 and 2010CB833605), the
Science and Technology Department Research Foundation of Hunan (2010SK3132) and the Ph.D. candidates' research innovation project of Hunan (CX2011B050), China.

\section{References}

1. El-Serag HB, Marrero JA, Rudolph L and Reddy KR: Diagnosis and treatment of hepatocellular carcinoma. Gastroenterology 134: 1752-1763, 2008.

2. Trinchet JC, Alperovitch A, Bedossa P, Degos F, Hainaut P and Beers BV: Epidemiology, prevention, screening and diagnosis of hepatocellular carcinoma. Bull Cancer 96: 35-43, 2009 (In French).

3. Debruyne EN and Delanghe JR: Diagnosing and monitoring hepatocellular carcinoma with alpha-fetoprotein: new aspects and applications. Clin Chim Acta 395: 19-26, 2008.

4. Singhal A, Jayaraman M, Dhanasekaran DN and Kohli V: Molecular and serum markers in hepatocellular carcinoma: predictive tools for prognosis and recurrence. Crit Rev Oncol Hematol 82: 116-140, 2012.

5. Leerapun A, Suravarapu SV, Bida JP, et al: The utility of Lens culinaris agglutinin-reactive alpha-fetoprotein in the diagnosis of hepatocellular carcinoma: evaluation in a United States referral population. Clin Gastroenterol Hepatol 5: 394-402, 2007.

6. Kobayashi M,Hosaka T,Ikeda K, et al: Highly sensitive AFP-L3\% assay is useful for predicting recurrence of hepatocellular carcinoma after curative treatment pre- and postoperatively. Hepatology Res 41: 1036-1045, 2011.

7. Joo M, Chi JG and Lee H: Expressions of HSP70 and HSP27 in hepatocellular carcinoma. J Korean Med Sci 20: 829-834, 2005.

8. Luk JM, Lam CT, Siu AF, et al: Proteomic profiling of hepatocellular carcinoma in Chinese cohort reveals heat-shock proteins (Hsp27, Hsp70, GRP78) up-regulation and their associated prognostic values. Proteomics 6: 1049-1057, 2006.

9. Shin E, Ryu HS, Kim SH, Jung H, Jang JJ and Lee K: The clinicopathological significance of heat shock protein 70 and glutamine synthetase expression in hepatocellular carcinoma. J Hepatobiliary Pancreat Sci 18: 544-550, 2011.

10. Tremosini S, Forner A, Boix L, et al: Prospective validation of an immunohistochemical panel (glypican 3, heat shock protein 70 and glutamine synthetase) in liver biopsies for diagnosis of very early hepatocellular carcinoma. Gut 61: 1481-1487, 2012.

11. Diehl JA, Yang W, Rimerman RA, Xiao H and Emili A: Hsc70 regulates accumulation of cyclin D1 and cyclin D1-dependent protein kinase. Mol Cell Biol 23: 1764-1774, 2003.

12. Calderwood SK, Khaleque MA, Sawyer DB and Ciocca DR: Heat shock proteins in cancer: chaperones of tumorigenesis. Trends Biochem Sci 31: 164-172, 2006.

13. Filmus J, Capurro M and Rast J: Glypicans. Genome Biol 9: 224, 2008.

14. Zittermann SI, Capurro MI, Shi W and Filmus J: Soluble Glypican 3 inhibits the growth of hepatocellular carcinoma in vitro and in vivo. Int J Cancer 126: 1291-1301, 2010.

15. Zittermann SI, Capurro MI, Shi W and Filmus J: Glypican-3 promotes the growth of hepatocellular carcinoma by stimulating canonical Wnt signaling. Cancer Res 65: 6245-6254, 2005.

16. Shirakawa H, Kumnuma T, Nishimura Y, et al: Glypican-3 is a useful diagnostic marker for a component of hepatocellular carcinoma in human liver cancer. Int J Oncol 34: 649-656, 2009.

17. Guido M, Roskams T, Pontisso P, et al: Squamous cell carcinoma antigen in human liver carcinogenesis. J Clin Pathol 61: 445-447, 2008.

18. Trerotoli P, Fransvea E, Angelotti U, et al: Tissue expression of squamous cellular carcinoma antigen (SCCA) is inversely correlated to tumor size in HCC. Mol Cancer 8: 29, 2009.

19. Giannelli G, Marinosci F, Sgarra C, Lupo L, Dentico P and Antonaci S: Clinical role of tissue and serum levels of SCCA antigen in hepatocellular carcinoma. Int J Cancer 116: 579-583, 2005.

20. Beneduce L, Castaldi F, Marino M, et al: Squamous cell carcinoma antigen-immunoglobulin $\mathrm{M}$ complexes as novel biomarkers for hepatocellular carcinoma. Cancer 103: 2558-2565, 2005.

21. Pontisso P, Quarta S, Caberlotto C, et al: Progressive increase of SCCA-IgM immune complexes in cirrhotic patients is associated with development of hepatocellular carcinoma. Int J Cancer 119: 735-740, 2006. 
22. Kladney RD, Bulla GA, Guo L, et al: GP73, a novel Golgilocalized protein upregulated by viral infection. Gene 249 : 53-65, 2000.

23. Zhang F, Gu Y, Li X, Wang W, He J and Peng T: Up-regulated Golgi phosphoprotein 2 (GOLPH2) expression in lung adenocarcinoma tissue. Clin Biochem 43: 983-991, 2010.

24. Fritzsche FR, Kristiansen G, Riener MO, Dietel M and Oelrich B: GOLPH2 expression may serve as diagnostic marker in seminomas. BMC Urol 10: 4, 2010.

25. Fritzsche FR, Riener MO, Dietel M, Moch H, Jung K and Kristiansen G: GOLPH2 expression in renal cell cancer. BMC Urol 8: 15, 2008.

26. Kristiansen G, Fritzsche FR, Wassermann K, et al: GOLPH2 protein expression as a novel tissue biomarker for prostate cancer: implications for tissue-based diagnostics. Br J Cancer 99: 939-948, 2008

27. Shi Y, Chen J, Li L, et al: A study of diagnostic value of golgi protein GP73 and its genetic assay in primary hepatic carcinoma. Technol Cancer Res Treat 10: 287-294, 2011.

28. Tian L, Wang Y, Xu D, et al: Serological AFP/Golgi protein 73 could be a new diagnostic parameter of hepatic diseases. Int J Cancer 129: 1923-1931, 2011.

29. Riener MO, Stenner F, Liewen H, et al: Golgi phosphoprotein 2 (GOLPH2) expression in liver tumors and its value as a serum marker in hepatocellular carcinomas. Hepatology 49: 1602-1609, 2009.

30. Zhou Y, Yin X, Ying J and Zhang B: Golgi protein 73 versus alpha-fetoprotein as a biomarker for hepatocellular carcinoma: a diagnostic meta-analysis. BMC Cancer 12: 17, 2012.

31. Mao YL, Yang HY, Xu HF, et al: Significance of Golgi glycoprotein 73, a new tumor marker in diagnosis of hepatocellular carcinoma: a primary study. Zhonghua Yi Xue Za Zhi 88: 948-951, 2008 (In Chinese).

32. Drake RR, Schwegler EE, Malik G, Diaz J, Block T, Mehta A and Semmes OJ: Lectin capture strategies combined with mass spectrometry for the discovery of serum glycoprotein biomarkers. Mol Cell Proteomics 5: 1957-1967, 2006.

33. Zhang Y, Deng ZS, Liao MM, Wang N, Zhang XQ, Yu HY and Zhang YD: Tumor associated glycoprotein-72 is a novel marker for poor survival in hepatocellular carcinoma. Pathol Oncol Res 18: 911-916, 2012.

34. Milenic DE, Brady ED, Garmestani K, Albert PS, Abdulla A and Brechbiel MW: Improved efficacy of alpha-particle-targeted radiation therapy: dual targeting of human epidermal growth factor receptor-2 and tumor-associated glycoprote in 72 . Cancer 116: 1059-1066, 2010.

35. Mracek T, Stephens NA, Gao D, et al: Enhanced ZAG production by subcutaneous adipose tissue is linked to weight loss in gastrointestinal cancer patients. Br J Cancer 104: 441-447, 2011.

36. Wang F, Geng Y, Zhang WM and Geng X: Identification of ZAG protein as a novel serologic biomarker candidate for liver cancer. Adv Mater Res 340: 383-389, 2012.

37. Naraki T, Kohno N, Saito H, Fujimoto Y, Ohhira M, Morita T and Kohgo Y: Gamma-carboxyglutamic acid content of hepatocellular carcinoma-associated des-gamma-carboxy prothrombin. Biochim Biophys Acta 1586: 287-298, 2002.

38. Nakamura S, Nouso K, Sakaguchi K, et al: Sensitivity and specificity of des-gamma-carboxy prothrombin for diagnosis of patients with hepatocellular carcinomas varies according to tumor size. Am J Gastroenterol 101: 2038-2043, 2006.

39. Baek YH, Lee JH, Jang JS, et al: Diagnostic role and correlation with staging systems of PIVKA-II compared with AFP. Hepatogastroenterology 56: 763-767, 2009.

40. Yamamoto K, Imamura $\mathrm{H}$, Matsuyama $\mathrm{Y}$, et al: Significance of alpha-fetopmtein and des-gamma-carbroxy prothrombin in patients with hepatocellular carcinoma undergoing hepatectomy. Ann Surg Oncol 16: 2795-2804, 2009.

41. Yamamoto K, Imamura H, Matsuyama Y, et al: AFP, AFP-L3, DCP, and GP73 as markers for monitoring treatment response and recurrence and as surrogate markers of clinicopathological variables of HCC. J Gastroenterol 45: 1272-1282, 2010.

42. Yao DF, Dong ZZ and Yao M: Specific molecular markers in hepatocellular carcinoma. Hepatobiliary Pancreat Dis Int 6: 241-247, 2007.

43. Cui R, He J, Zhang F, et al: Diagnostic value of protein induced by vitamin $\mathrm{K}$ absence (PIVKAII) and hepatoma-specific band of serum gamma-glutamyl transferase (GGTII) as hepatocellular carcinoma markers complementary to alpha-fetoprotein. Br J Cancer 88: 1878-1882, 2003.
44. Wang CS, Lin CL, Lee HC, et al: Usefulness of serum desgamma-carboxy prothrombin in detection of hepatocellular carcinoma. World J Gastroenterol 11: 6115-6119, 2005.

45. Tangkijvanich $\mathrm{P}$, Tosukhowong $\mathrm{P}$, Bunyongyod $\mathrm{P}$, et al: Alpha-L-fucosidase as a serum marker of hepatocellular carcinoma in Thailand. Southeast Asian J Trop Med Public Health 30: 110-114, 1999.

46. Fawzy Montaser M, Amin Sakr M and Omar Khalifa M: Alpha-L-fucosidase as a tumour marker of hepatocellular carcinoma. Arab J Gastroenterol 13: 9-13, 2012.

47. Pillai AA and Fimmel CJ: Emerging serum biomarkers of HCC. Clin Gastroenterol 27: 247-262, 2012.

48. Zhou L, Liu J and Luo F: Serum tumor markers for detection of hepato-cellular carcinoma. World J Gastroenterol 12: 1175-1181, 2006.

49. Balzarini P, Benetti A, Invernici G, et al: Transforming growth factor-betal induces microvascular abnormalities through a down-modulation of neural cell adhesion molecule in human hepatocellular carcinoma. Lab Invest 92: 1297-1309, 2012.

50. Dong ZZ, Yao DF, Yao M, et al: Clinical impact of plasma TGF-beta1 and circulating TGF-beta1 mRNA in diagnosis of hepatocellular carcinoma. Hepatobiliary Pancreat Dis Int 7: 288-295, 2008

51. Xiang ZL, Zeng ZC, Fan J, et al: Gene expression profiling of fixed tissues identified hypoxia-inducible factor-1 $\alpha$, VEGF, and matrix metalloproteinase-2 as biomarkers of lymph node metastasis in hepatocellular carcinoma. Clin Cancer Res 17: 5463-5472, 2011.

52. Zhang L, Wang JN, Tang JM, et al: VEGF is essential for the growth and migration of human hepatocellular carcinoma cells. Mol Biol Rep 39: 5085-5093, 2012.

53. Zhi H, Zhan J, Deng QL, et al: Postoperative detection of AFP mRNA in the peripheral blood of hepatic cellular carcinoma patients and its correlation with recurrence. Zhonghua Zhong Liu Za Zhi 29: 112-115, 2007 (In Chinese).

54. Yamamoto Y, Kosaka N, Tanaka M, et al: MicroRNA-500 as a potential diagnostic marker for hepatocellular carcinoma. Biomarkers 14: 529-538, 2009.

55. Xiong Y, Fang JH, Yun JP, et al: Effects of microRNA-29 on apoptosis, tumorigenicity, and prognosis of hepatocellular carcinoma. Hepatology 51: 836-845, 2010.

56. Hou J, Lin L, Zhou W, et al: Identification of miRNomes in human liver and hepatocellular carcinoma reveals miR-199a/b-3p as therapeutic target for hepatocellular carcinoma. Cancer Cell 19: 232-243, 2011.

57. Xu Y, Xia F, Ma L, Shan J, et al: MicroRNA-122 sensitizes HCC cancer cells to adriamycin and vincristine through modulating expression of MDR and inducing cell cycle arrest. Cancer Lett 310: 160-169, 2011

58. Volinia S, Calin GA, Liu CG, et al: A microRNA expression signature of human solid tumors defines cancer gene targets. Proc Natl Acad Sci USA 103: 2257-2261, 2006.

59. Medina PP, Nolde M and Slack FJ: OncomiR addiction in an in vivo model of microRNA-21-induced pre-B-cell lymphoma. Nature 467: 86-90, 2010.

60. Meng F, Henson R, Wehbe-Janek H, Ghoshal K, Jacob ST and Patel T: MicroRNA-21 regulates expression of the PTEN tumor suppressor gene in human hepatocellular cancer. Gastroenterology 133: 647-58, 2007.

61. Tomimaru Y, Eguchi H, et al: Circulating microRNA-21 as a novel biomarker for hepatocellular carcinoma. J Hepatol 56: 167-175, 2012.

62. Jin ZH, Yang RJ, Dong B and Xing BC: Progenitor gene DLK1 might be an independent prognostic factor of liver cancer. Expert Opin Biol Ther 8: 371-377, 2008.

63. Liu Y, Li Y, Guo F, et al: Identification of HTA as a novel-specific marker for human hepatocellular carcinoma. J Cancer Res Clin Oncol 136: 1187-1192,2010.

64. Xieraili M, Yasen M, Mogushi K, et al: Villin 1 is a predictive factor for the recurrence of high serum alpha-fetoprotein-associated hepatocellular carcinoma after hepatectomy. Cancer Sci 103: 1493-1501, 2012. 